\title{
Endoscopic Treatment of Iatrogenic Perforation of Sigmoid Diverticulum: A Case Report of Multidisciplinary Management
}

\author{
Giacomo Emanuele Maria Rizzo ${ }^{1,3}$, Giuseppina Ferro ${ }^{3}$, Giovanna Rizzo ${ }^{2,3}$, Giovanni Di Carlo ${ }^{3}$, Alessandro Cantone ${ }^{3}$, Gaetano \\ Giuseppe Di Vita ${ }^{2}$, and Carmelo Sciumè $\dot{e}^{2,3}$ \\ ${ }^{1}$ Section of Gastroenterology \& Hepatology, Department of Health Promotion Sciences Maternal and Infant Care, Internal Medicine \\ and Medical Specialties, PROMISE, University of Palermo, Palermo, ${ }^{2}$ Department of Surgical, Oncological and Oral Science, Palermo \\ University Hospital, Palermo, ${ }^{3}$ Section of Endoscopy, Department of General Surgery, San Giovanni di Dio Hospital, Agrigento, Italy
}

Iatrogenic perforations are severe complications of gastrointestinal endoscopy; therefore, their management should be adequately planned. A 77-year-old man with a history of diverticulosis underwent a colonoscopy for anemia. During the procedure, an iatrogenic perforation occurred suddenly in the sigmoid colon, near a severe angle among the numerous diverticula. Through-thescope clips were immediately applied to treat it and close mucosal edges. Laboratory tests showed increased levels of inflammation and infection, and although there were no complaints of abdominal pain, the patient had an extremely distended abdomen. A multidisciplinary board began management based on a conservative approach. Pneumoperitoneum was treated with computed tomography-assisted drainage. After 72 hours, his intestinal canalization and laboratory tests were normal. Though this adverse event is rare, a multidisciplinary board should be promptly gathered upon occurrence, even if the patient appears clinically stable, to consider a conservative approach and avoid surgical treatment. Clin Endosc 2022;55:292-296

Key Words: Colonoscopy; Diverticulum; Endoscopy; Intestinal perforation

\section{INTRODUCTION}

Iatrogenic perforations (IPs) are rare and severe complications of diagnostic and therapeutic gastrointestinal (GI) endoscopy; therefore, immediate and adequate management should be planned. Acute IP related to GI endoscopy is defined as the recognition of gas or luminal fluids outside the GI $\operatorname{tract}^{1}$ or any endoscopically-identified, definite visible sign of perforation during or related to endoscopy. ${ }^{2}$ Usually, perforation occurs in advanced endoscopic procedures that are invasive and have a higher risk than diagnostic endoscopy (endoscopic submucosal dissection, large endoscopic mucosal resection,

\footnotetext{
Received: November 19, 2020 Revised: January 30, 2021

Accepted: February 8, 2021

Correspondence: Carmelo Sciumè

Department of Surgical, Oncological and Oral Science, University of Palermo, Palermo 90127, Italy

Tel: +39-91-655-1111, Fax: +39-91-655-2119, E-mail: carmelo.sciume@unipa.it ORCID: https://orcid.org/0000-0002-7641-8273
}

(c) This is an Open Access article distributed under the terms of the Creative Commons Attribution Non-Commercial License (http://creativecommons.org/ licenses/by-nc/3.0) which permits unrestricted non-commercial use, distribution, and reproduction in any medium, provided the original work is properly cited. peroral endoscopic myotomy, and large balloon dilation in the papilla). ${ }^{3-6}$ Nevertheless, IP during elective colonoscopy is also associated with a high risk of morbidity and mortality. ${ }^{7}$ In fact, when this complication occurs, physicians usually immediately send the patient to surgery instead of considering the endoscopic treatment as definitive. Perforations in the sigmoid colon are mostly treated with sigmoid resection, and this usually happens because of the physician's fear of delaying surgery too long; thus, an increase in morbidity and mortality are surgery-related. In contrast, an IP should be managed by a multidisciplinary approach, which includes a board of endoscopists, radiologists, and surgeons. Moreover, to reduce mortality and improve outcomes, the multidisciplinary board needs to meet as soon as possible with physicians with expertise. Guidelines help handle these adverse events; however, because of the rarity of this event, literature requires reports of clinical cases to demonstrate proper management.

We present the case of a patient with an IP of a diverticulum in the sigmoid colon that occurred during an elective colonoscopy; the patient was endoscopically treated, and a multidisciplinary approach was planned for his treatment. 


\section{CASE REPORT}

A 77-year-old Italian man with a history of diverticulosis was admitted to our hospital for a diagnostic colonoscopy for mild anemia (hemoglobin $10.5 \mathrm{~g} / \mathrm{L}$ ). He did not take any immunomodulatory or anticoagulation drugs; however, he took an anti-platelet drug (aspirin $100 \mathrm{mg}$ once a day) for primary prophylaxis. He had no comorbidities. Diagnostic colonoscopy with carbon dioxide insufflation was performed by an expert endoscopist with an experience of more than 800 colonoscopies/year. Examination showed a clean intestinal lumen without fecal residue and normotrophic mucosa. Moreover, his sigmoid colon was found to have more than fifteen diverticula with a rigid lumen, corresponding to Diverticular Inflammation and Complication Assessment classification II. ${ }^{8}$ An IP of about 8-9 mm suddenly occurred in a diverticulum of the sigmoid colon, showing peritoneal breach in the colic

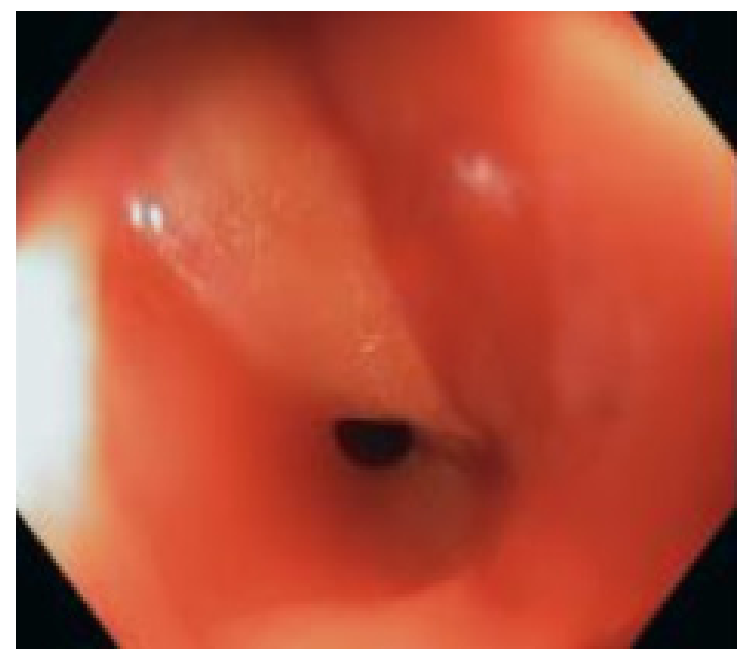

Fig. 1. Endoscopic view of the iatrogenic perforation. Colonoscopy shows the breach in diverticulum of sigmoid colon. The breach is characterized by an hole and peritoneal tissue.
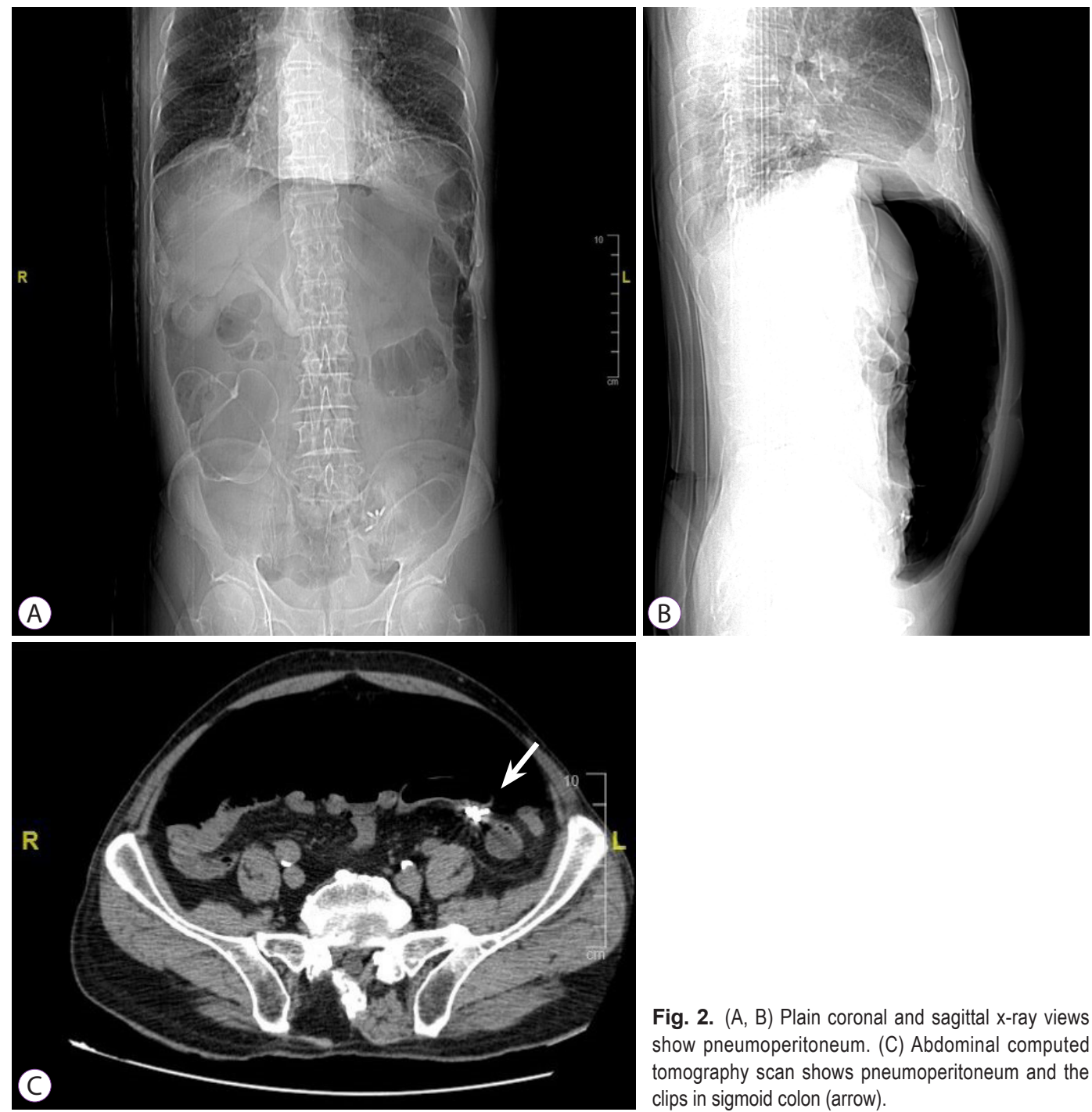

Fig. 2. (A, B) Plain coronal and sagittal $x$-ray views show pneumoperitoneum. (C) Abdominal computed tomography scan shows pneumoperitoneum and the clips in sigmoid colon (arrow). 

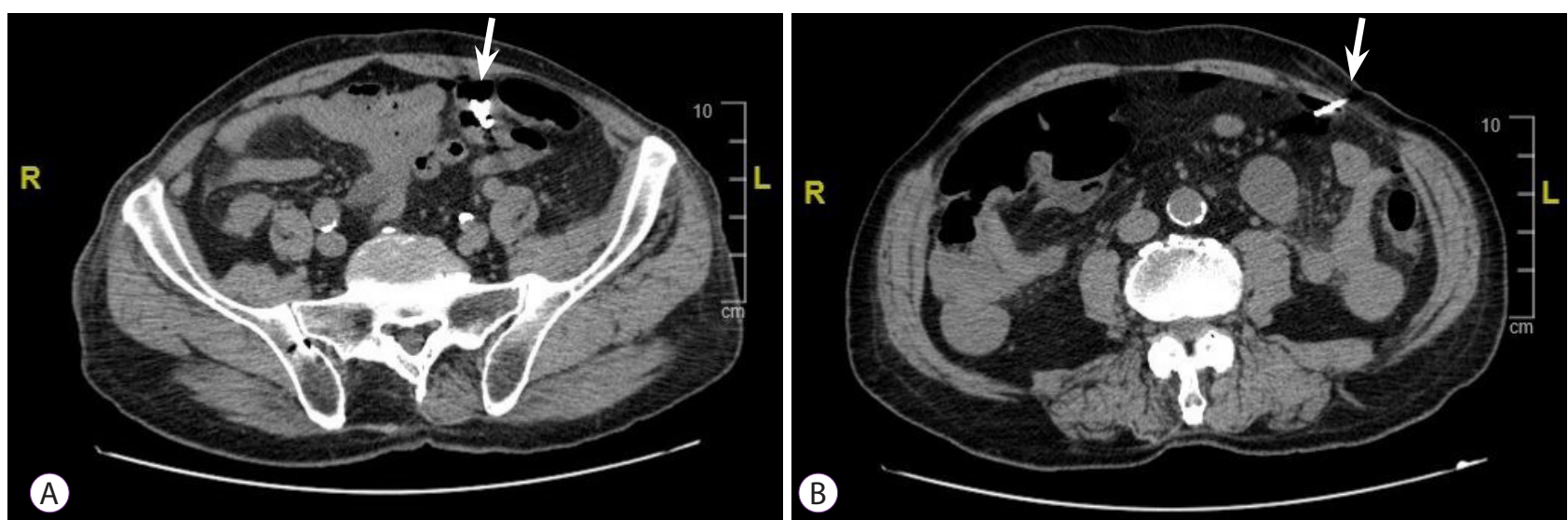

Fig. 3. Abdominal computed tomography scan after drainage of pneumoperitoneum. (A) View of clips in the sigmoid colon (arrow). (B) Point of insertion of Veres needle (arrow).

mucosa (Fig. 1). IP occurred due to a combination of events, including direct mechanical trauma caused by the movement of the tip of the colonoscope in the hole of the diverticulum during insertion and the presence of a weaker and pathological area (diverticula and rigidity of the lumen). Immediately, the breach was closed using four through-the-scope (TTS) clips, closing the mucosal edge. During the next 3 hours, the patient did not complain of abdominal pain, but only discomfort and bloating, despite an extremely distended abdomen. Therefore, he was hospitalized to monitor the clinical course. Urgent laboratory tests (within 3 hours) showed an increase in white blood cell count (WBC, $12500 / \mathrm{mm}^{3}$ ), with $88 \%$ neutrophils, $149 \mathrm{mg} / \mathrm{L}$ C-reactive protein (CRP), and $2.28 \mathrm{ng} / \mathrm{mL}$ procalcitonin. Although the patient met the criteria for sepsis, he was hemodynamically stable with a heart rate of 80 beats per minute and blood pressure of 123/72 mmHg; hence, it was unclear whether the surgical approach was still necessary. A computer tomography (CT) scan was performed, showing massive pneumoperitoneum without any other sign of peritonitis (Fig. 2). However, a multidisciplinary board composed of endoscopists, radiologists, and surgeons analyzed the patient's condition due to the possibility of immediately undergoing surgery despite endoscopic treatment. Ultimately, management was established and based on a conservative approach with a wait-and-see strategy: fasting and intravenous alimentation; antibiotic treatment with a combination of piperacillin/ tazobactam, fluoroquinolones, and metronidazole; and continuous monitoring of clinical, laboratory, and vital signs. Between 12 and 24 hours later, despite stable vital signs, no fever, and a slight improvement in laboratory tests (WBC, 11000/ $\mathrm{mm}^{3}$; $85 \%$ neutrophils; CRP, $142 \mathrm{mg} / \mathrm{L}$; and procalcitonin $1.81 \mathrm{ng} / \mathrm{mL}$ ), the patient developed moderate abdominal pain, probably secondary to massive pneumoperitoneum, which had not decreased in volume. Therefore, it was drained with CT assistance using a spring-loaded needle (Veres needle) to reduce distension and avoid development of compartment syndrome (Fig. 3). After 48 hours, the patient did not complain of any discomfort or other abdominal signs; therefore, he started oral liquid alimentation, following the 2017 World Society of Emergency Surgery recommendation. ${ }^{9}$ In 72 hours, laboratory tests showed further improvement (WBC, 9500/ $\mathrm{mm}^{3}$; neutrophils, $85 \%$; CRP, $60 \mathrm{mg} / \mathrm{L}$; procalcitonin, $1.17 \mathrm{ng} /$ $\mathrm{mL}$ ) with a stable clinical course and normal intestinal canalization; therefore, the patient was discharged and continued antibiotic treatment at home. A one-month follow-up showed good health, normal canalization, and laboratory tests.

\section{DISCUSSION}

Colonoscopy-induced perforation (CIP) is a major complication with an incidence ranging from $0.06 \%-0.8 \%{ }^{10,11,12}$ The first choice of treatment has always been surgery when peritonitis or fecal intraperitoneal materials are demonstrated. ${ }^{13}$ Nowadays, a proper stratification of patients is essential because peritonitis does not always occur and endoscopic treatment may be effective in some cases of IP (successful rate of $83.3 \%$ when perforation was immediately visualized by the endoscopist). ${ }^{2,11}$ Because of the rarity of IP during a diagnostic colonoscopy, we present how a conservative approach may be considered in a case of perforation during a diagnostic colonoscopy. In fact, a conservative and wait-and-see strategy may avoid surgical treatment, reducing the risk of surgery and improving mortality and outcomes. However, it is essential to 
properly stratify patients before choosing between surgery and endoscopy. Multivariate analysis showed that when IP occurs, old age (hazard ratio [HR], 6.603; 95\% CI, 1.013-43.063), an American Society for Anesthesiologists score $\geq 3$ (HR, 7.497; $95 \% \mathrm{CI}, 1.187-47.363$ ), and conversion from non-surgical to surgical procedures (HR, 33.204; 95\% CI, 4.230-260.617) were independent predictors of poor outcomes (mortality and morbidity). Moreover, a perforation size $>15 \mathrm{~mm}$ was a significant predictor for conversion. ${ }^{10}$ Hence, it is important to avoid conversion, giving the proper indication from the beginning. As mentioned above, patients with peritonitis signs are immediately treated with surgery (usually with primary closure or bowel resection). In a retrospective, 16-year experience study, IP and consequent peritonitis after diagnostic colonoscopy mostly occurred in the sigmoid colon ( $76 \%$ of cases).$^{14}$ All patients in this study had surgical indications, but the less invasive laparoscopic-assisted surgical treatment was performed in only $32 \%$ of patients, while open surgery and open conversion was necessary in $60 \%$ and $8 \%$ of them, respectively, increasing surgery-related morbidity. Complications occurred in 36\% of cases, with $8 \%$ re-perforation and $0 \%$ mortality, showing that surgery was effective in these patients. Our patient had good bowel preparation, no leakage of fluid or fecal content on CT scan, no signs of peritoneal irritation, a perforation size of $<15$ $\mathrm{mm}$, and immediate identification of the perforation site. Our case supports the use of TTS clips as a reasonable choice in this setting if the perforation is less than $10-15 \mathrm{~mm}$, and OverThe-Scope-Clips (OTSC) may be used as well. ${ }^{15-16}$ The decision-making process is complicated, and the decision of our endoscopist's team was based on the size of the perforation, its inconvenient location (inside a diverticulum among diverticula in a rigid sigmoid colon), and the endoscopist's confidence in using TTS clips. Moreover, the OTSC system requires withdrawal of the endoscope and re-insertion after assembling the clipping system, which prolongs procedure time and makes it more difficult to relocate the IP, even if it is in the sigmoid colon. Another complicated point concerns risk factors for surgery within 24 hours after endoscopic clip closure. Cho et $\mathrm{al}^{17}$ showed large perforation (odds ratio [OR], 9.25), severe abdominal pain (OR, 4.30), fever (OR, 5.05), leukocytosis (OR, 6.58), and a large amount of free air in the peritoneum (OR, $4.05)$ to be risk factors. In our case, the patient had a small perforation, no fever, and only initial leukocytosis, while the abdominal pain and free air in the peritoneum disappeared completely after drainage of the pneumoperitoneum. The drainage of pneumoperitoneum was debated because of the use of carbon dioxide insufflation during colonoscopy, which is known to be safer and absorbed faster ${ }^{18}$ than normal air in the case of perforation, resulting in reduction of the secondary deleterious effects of perforation. ${ }^{19,20}$ The multidisciplinary board chose the timing and strategy of treatment based on current literature. ${ }^{2.9}$ Therefore, we continuously examined clinical signs and symptoms and serial blood samples, which showed a slight improvement, and we performed drainage between 12 and 24 hours of perforation because of the persistence of a high volume of intra-abdominal gas and abdominal pain, resulting in resolution of the latter.

In conclusion, we recommend a multidisciplinary approach in the cases of CIP in which endoscopic treatment may achieve a successful outcome. It is essential to properly identify patients who may benefit from a conservative treatment and continuously monitor their clinical, laboratory, and hemodynamic parameters to avoid delays in conversion to surgical treatment.

Conflicts of Interest

The authors have no potential conflicts of interest.

Funding

None.

Author Contributions

Conceptualization: Giacomo Emanuele Maria Rizzo

Data curation: GEMR

Formal analysis: GEMR

Methodology: GEMR, Giovanna Rizzo

Project administration: GEMR, CS

Resources: Giuseppina Ferro, GR

Software: Giovanni di Carlo, Alessandro Cantone, GF

Supervision: Carmelo Sciumè

Validation: CS

Visualization: GF, GDC, CS

Writing-original draft: GEMR

Writing-review \& editing: GF, GR, Gaetano Giuseppe Di Vita, CS

\section{ORCID}

Giacomo Emanuele Maria Rizzo https://orcid.org/0000-0001-9335-6740 Giuseppina Ferro

Giovanna Rizzo

Giovanni Di Carlo

Alessandro Cantone

Gaetano Giuseppe Di Vita

Carmelo Sciumè https://orcid.org/0000-0002-8106-1336 https://orcid.org/0000-0002-4204-0735 https://orcid.org/0000-0003-4891-5958 https://orcid.org/0000-0001-6953-6127 https://orcid.org/0000-0003-0311-3089 https://orcid.org/0000-0002-7641-8273

\section{REFERENCES}

1. Raju GS, Saito Y, Matsuda T, Kaltenbach T, Soetikno R. Endoscopic management of colonoscopic perforations (with videos). Gastrointest Endosc 2011;74:1380-1388.

2. Paspatis GA, Arvanitakis M, Dumonceau JM, et al. Diagnosis and management of iatrogenic endoscopic perforations: European Society of Gastrointestinal Endoscopy (ESGE) Position Statement - Update 2020. Endoscopy 2020;52:792-810.

3. Toyonaga T, Man-i M, East JE, et al. 1,635 Endoscopic submucosal dissection cases in the esophagus, stomach, and colorectum: complication rates and long-term outcomes. Surg Endosc 2013;27:1000-1008. 
4. Paspatis GA, Konstantinidis K, Tribonias G, et al. Sixty- versus thirty-seconds papillary balloon dilation after sphincterotomy for the treatment of large bile duct stones: a randomized controlled trial. Dig Liver Dis 2013;45:301-304

5. Mangiavillano B, Caruso A, Manta R, et al. Over-the-scope clips in the treatment of gastrointestinal tract iatrogenic perforation: A multicenter retrospective study and a classification of gastrointestinal tract perforations. World J Gastrointest Surg 2016;8:315-320.

6. Jayanna M, Burgess NG, Singh R, et al. Cost analysis of endoscopic mucosal resection vs surgery for large laterally spreading colorectal lesions. Clin Gastroenterol Hepatol 2016;14:271-278.e82.

7. Martínez-Pérez A, de’Angelis N, Brunetti F, et al. Laparoscopic vs. open surgery for the treatment of iatrogenic colonoscopic perforations: a systematic review and meta-analysis. World J Emerg Surg 2017;12:8.

8. Tursi A, Brandimarte G, Di Mario F, et al. Development and validation of an endoscopic classification of diverticular disease of the colon: the DICA classification. Dig Dis 2015;33:68-76.

9. de'Angelis N, Di Saverio S, Chiara O, et al. 2017 WSES guidelines for the management of iatrogenic colonoscopy perforation. World J Emerg Surg 2018;13:5.

10. An SB, Shin DW, Kim JY, Park SG, Lee BH, Kim JW. Decision-making in the management of colonoscopic perforation: a multicentre retrospective study. Surg Endosc 2016;30:2914-2921.

11. Derbyshire E, Hungin P, Nickerson C, Rutter MD. Colonoscopic perforations in the English National Health Service Bowel Cancer Screening Programme. Endoscopy 2018;50:861-870.
12. Mai CM, Wen CC, Wen SH, et al. Iatrogenic colonic perforation by colonoscopy: a fatal complication for patients with a high anesthetic risk. Int J Colorectal Dis 2010;25:449-454.

13. Carpio G, Albu E, Gumbs MA, Gerst PH. Management of colonic perforation after colonoscopy. Report of three cases. Dis Colon Rectum 1989;32:624-626.

14. Lim DR, Kuk JK, Kim T, Shin EJ. The analysis of outcomes of surgical management for colonoscopic perforations: a 16-years experiences at a single institution. Asian J Surg 2020;43:577-584.

15. Raju GS. Endoscopic clip closure of gastrointestinal perforations, fistulae, and leaks. Dig Endosc 2014;26(Suppl 1):95-104.

16. Jung Y. Endoscopic management of iatrogenic colon perforation. Clin Endosc 2020;53:29-36.

17. Cho SB, Lee WS, Joo YE, et al. Therapeutic options for iatrogenic colon perforation: feasibility of endoscopic clip closure and predictors of the need for early surgery. Surg Endosc 2012;26:473-479.

18. Saltzman HA, Sieker HO. Intestinal response to changing gaseous environments: normobaric and hyperbaric observations. Ann NY Acad Sci 1968;150:31-39.

19. ASGE Technology Committee, Lo SK, Fujii-Lau LL, et al. The use of carbon dioxide in gastrointestinal endoscopy. Gastrointest Endosc 2016;83:857-865

20. Memon MA, Memon B, Yunus RM, Khan S. Carbon dioxide versus air insufflation for elective colonoscopy: a meta-analysis and systematic review of randomized controlled trials. Surg Laparosc Endosc Percutan Tech 2016;26:102-116. 